unter den Grundannahmen, die ich festgelegt habe, kaum besser machen. Das ist bewiesenermaßen so, und alle anderen Möglichkeiten sind weniger gut. Auch wenn Lieferungen immer zu spät kommen, Fahrer immer krank werden, auch wenn wir irgendwo immer das Rauschen der Welt, immer eine Abweichung haben - am Ende ist dieses Modell das beste. Dafür gibt es einen Beweis. Auf diesem Fundament stehe ich und insofern ist Mathematik für mich Berufung.

Wie können sich Mathematik-Studierende vorbereiten, wenn Sie sich für die Start-up-Branche interessieren?

Ich kann jedem nur raten, schon als Werkstudent aktiv zu werden. Das war jedenfalls für mich der Schlüssel. Man hat die Chance, etwas auszuprobieren, kann erst mal irgendwo anfangen, und selbst wenn es einem nach einem halben Jahr nicht mehr gefällt, nimmt einem das keiner übel. Danach ist man auf jeden Fall schlauer. Die Unternehmen haben auch etwas davon, denn Werkstudenten sind günstige, gut qualifizierte Arbeitskräfte. Foodora und auch andere Start-ups stellen laufend Werkstudenten ein. Hier in Berlin gibt es bei diesen Unternehmen viele Möglichkeiten für junge Mathematiker. Auch in den kommenden Jahren.

Kristina Vaillant ist freie Journalistin in Berlin und arbeitet regelmäBig für das Medienbüro der Deutschen Mathematiker-Vereinigung. http://www.vaillant-texte.de

\title{
Mathematikstudium mal anders
}

Leoni Winschermann

Es gibt etwas, das mich von den meisten anderen deutschen Mathematikstudenten unterscheidet: Ich studiere in den Niederlanden! Viele Leute stutzen schon, wenn man nur fallen lässt, dass man sich freiwillig mit Mathematik auseinandersetzt. Aber selbst Freunde des Faches werden skeptisch, wenn ich erzähle, wo ich studiere. Der Studiengang ist doch zulassungsfrei? Ist es dann nicht logischer in Deutschland zu bleiben? Was treibt einen dann ins Ausland?

Zunächst einmal muss man sich dennoch bewusst machen, dass das Auslandsstudium eine Medaille mit zwei Seiten ist. Der größte Nachteil sind die nicht unerhebli- chen Studiengebühren. In den Niederlanden belaufen diese sich auf beinahe 2000 Euro pro Studienjahr. Was jeder Student zudem als erstes lernt: Die benötigten Bücher sind teuer. Hinzu kommen in der Regel noch Unterhaltskosten und Mietzahlungen. Das ist viel Geld. Vor allem wenn man auf die Unterstützung der Eltern angewiesen ist und vielleicht noch Geschwister hat. Dann muss man sich also durchaus mit Finanzierungsmöglichkeiten auseinandersetzen. Der passende Nebenjob, der einen über Wasser halten könnte, ist vor allem im ersten Studienjahr viel zu zeitaufwendig. Stattdessen empfehle ich es, sich mit dem für einen Antrag auf Auslands-Bafög not-
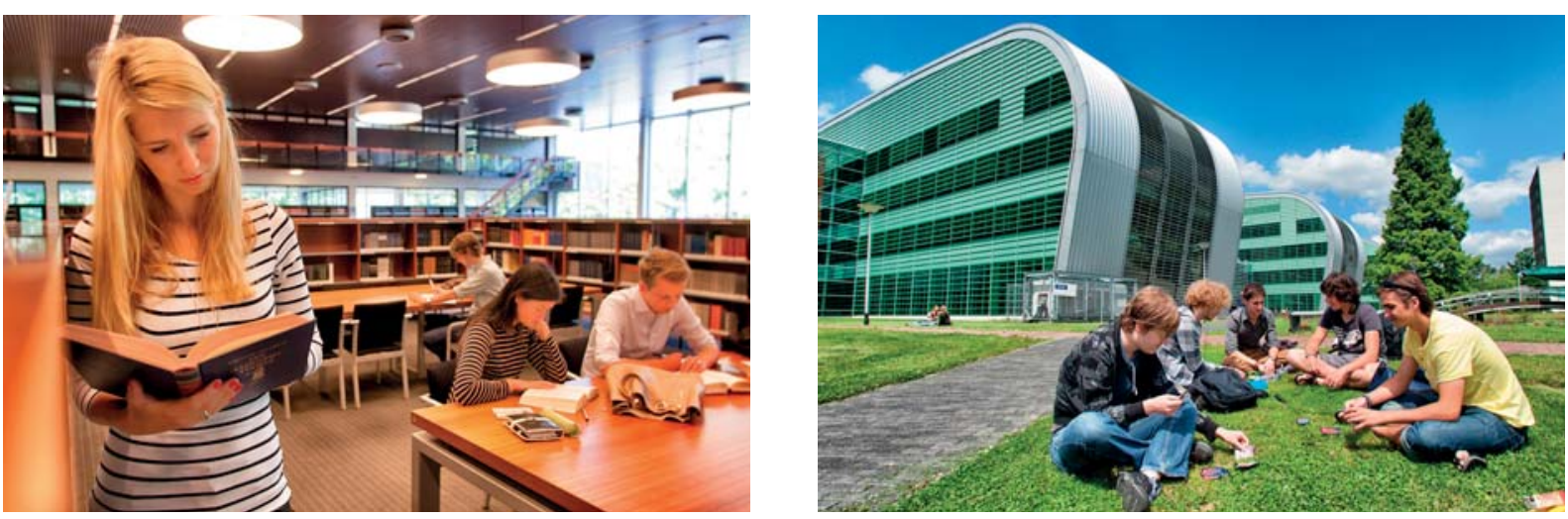

Links: In jedem Gebäude bietet eine Fachbibliothek die Möglichkeit, sich seinen Studien zu widmen. Zudem gibt es eine allgemeine Universitätsbibliothek, die erst neulich modernisiert worden ist. Rechts: Das Huygensgebouw ist das Gebäude, das die Naturwissenschaften beherbergt. Hier befinden sich sowohl die Hörsäle als auch die Arbeitsplätze der Professoren. Forschung und Lehre gehören untrennbar zusammen. (Fotos: Beeldredactie Radboud University) 
wendigen Papierkram herumzuschlagen. Gerade im ersten Jahr lohnt sich der Aufwand, denn dann hat man die Möglichkeit, die anfallenden Studiengebühren erstattet zu bekommen. Auch werden heute mehr Stipendien denn je vergeben. Dabei kommt es nicht nur auf die akademischen Leistungen an. Es werden auch Stipendien speziell an Arbeiterkinder, weibliche Studenten oder gesellschaftlich besonders engagierte Jugendliche vergeben, um nur ein paar zu nennen. Viele von diesen Stipendien lassen sich ins Ausland „mitnehmen“. Eine Recherche zahlt sich also aus.

Uns Mathematikern wird oft ein fehlendes Talent für Fremdsprachen nachgesagt. Wenn man allerdings ins Ausland geht, ist die Chance, dass die Unterrichtssprache Deutsch ist, verschwindend gering. In meinem Fall werden alle Veranstaltungen des Studiengangs auf Niederländisch abgehalten. Nun ist es in der Regel nicht so, dass man als Abiturient rein zufällig ausreichende Sprachkenntnisse vorweisen kann. Vor allem fernab der Grenze ist Niederländisch keine geläufige Sprache. Allerdings bietet sich hier den nicht vollkommen Sprachunbegabten unter uns eine echte Chance: Die meisten Universitäten in den Niederlanden bieten speziell für deutsche Studenten Intensivsprachkurse an. Durch die Ähnlichkeiten zwischen Deutsch und Niederländisch ist es nämlich durchaus machbar innerhalb von fünf Wochen ein angemessenes Niveau zu erreichen. Hier könnte man wieder auf den Kostenaspekt zurückkommen. An meiner Uni gibt es hierzu eine Lösung. Die Kosten des Kurses werden erstattet, wenn man das erste Jahr des Studiums besteht.

Hier drängt sich schon wieder eine Frage auf: Was bedeutet es denn, ein Studienjahr zu „bestehen“? Bei uns in den Niederlanden gibt es für keinen einzigen Studiengang einen Numerus Clausus in diesem Sinne. Was es sehr wohl gibt, ist die bindende Studienempfehlung. Das bedeutet, dass man in seinem ersten Studienjahr mindestens drei Viertel der zu erreichenden Studienpunkte bekommen muss. Schafft man das nicht, muss man den Studiengang verlassen. In diesem System sehe ich einen Vorteil gegenüber dem an deutschen Unis. Auf diese Weise findet man im ersten Jahr heraus, ob man dem Studium gewachsen ist. Für die folgenden Jahre nimmt der Druck demnach etwas ab, da man dann keine solche Mindestanforderung mehr erfüllen muss. Außerdem ist diese Hürde für motivierte Studenten, die etwas Zeit und Arbeit zu investieren bereit sind, leicht zu überwinden.

Dennoch ist das Studium der Mathematik selbst für interessierte Studenten oft noch eine ziemliche Herausforderung. Es hat eine der höchsten Abbrecherquoten in ganz Deutschland. Sicherlich liegt das auch am Unterschied zwischen Schulmathematik und universitärer Mathematik. Aber auch der abstrakte Charakter der Disziplin ist nicht ganz unschuldig. Man kann also einen generell hohen Schwierigkeitsgrad feststellen. Ist es dann überhaupt ein realistisches Ziel, diesen Studiengang in einer Fremdsprache zu meistern, die man vielleicht erst seit einigen

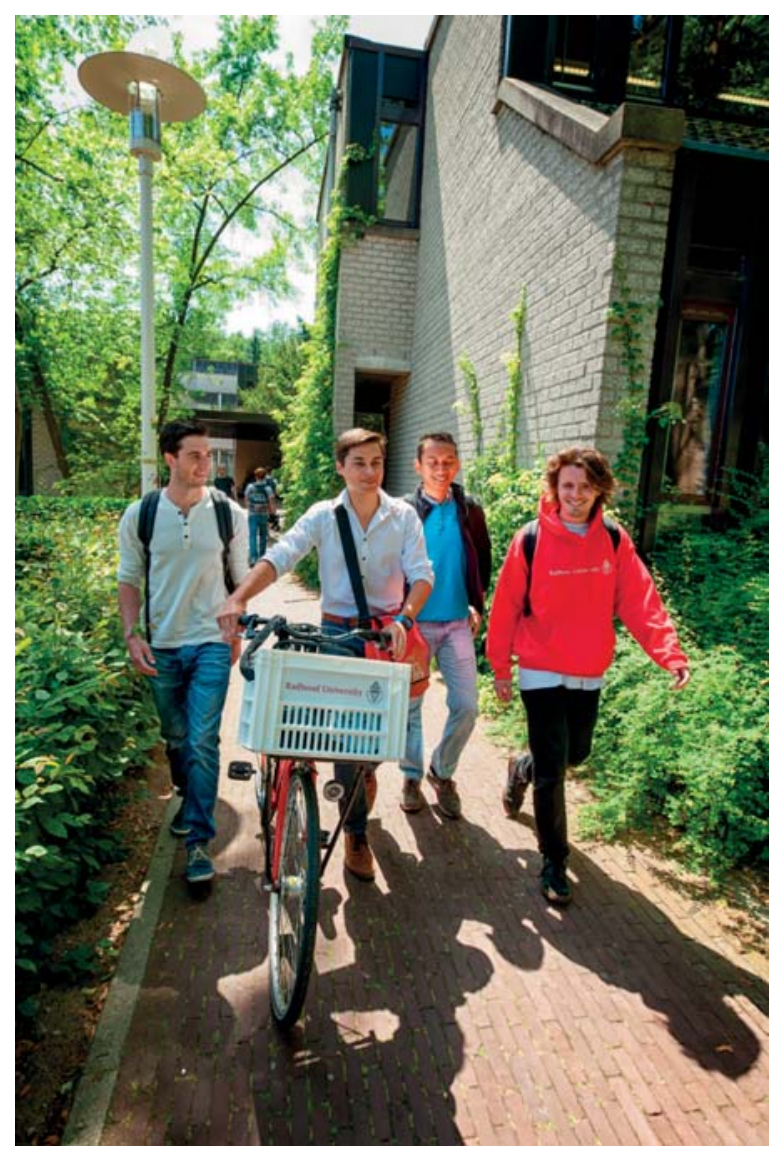

Das Fahrrad darf auf einem niederländischen Unicampus natürlich nicht fehlen. (Foto: Beeldredactie Radboud University)

Wochen spricht? Erstaunlicherweise lautet die Antwort ,ja“. Ich bin zwei Monate vor Studienbeginn erstmalig mit dem Niederländischen in Berührung gekommen. Dennoch saß ich im letzten September in meiner ersten Vorlesung und konnte den Worten des Dozenten folgen. Innerhalb der ersten paar Wochen eignet man sich sein ganz eigenes Fachvokabular an. Ziemlich bald erreicht man dann auch den Punkt, an dem man den Unterschied gar nicht mehr wahrnimmt. Was dabei auch sehr hilft: Es gibt nicht sehr viele deutsche Mathematikstudenten an niederländischen Universitäten. Man baut also zwangsläufig Freundschaften mit Niederländern auf. Das unterstützt die Sprachentwicklung natürlich immens.

Schön und gut. Man kann also alle Hürden auf die eine oder andere Art überwinden. Doch warum genau sollte man überhaupt in den Niederlanden studieren? Zunächst muss man sich noch einmal mehr bewusst machen, dass auch die Niederlande, obwohl sie unser direkter Nachbar sind, in die Kategorie „Ausland“ fallen. Das erste Argument, das man für ein Studium dort finden kann, ist also der Lebenslauf. Auslandsaufenthalte, auch im Rahmen eines Studiums, werden bei Bewerbungen immer positiv angerechnet. Aber bietet ein solcher Aufenthalt tatsächlich einen Mehrwert? Was macht ihn so besonders? Ein wichtiger Aspekt ist meiner Meinung nach, dass man auf diese Weise seine Selbstständigkeit ganz anders unter 


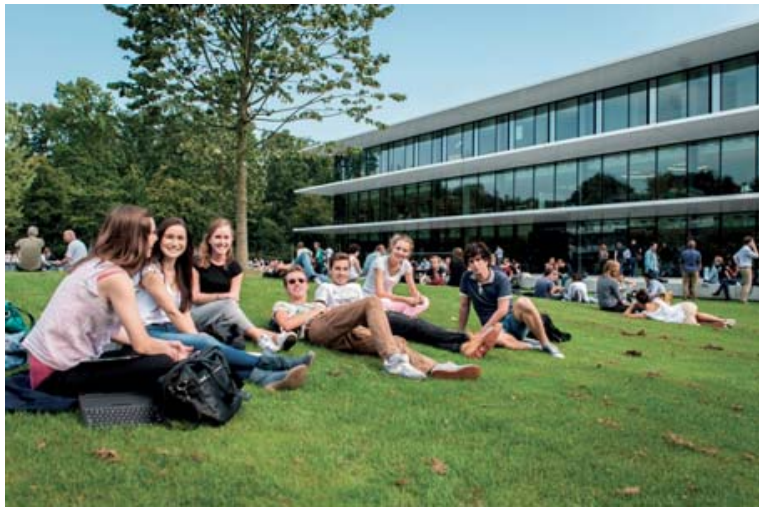

Der grüne Campus - ein weiteres Plus der Radboud. Zwischen den Vorlesungen gemütlich im Gras zu liegen oder in den benachbarten Park zu gehen, hilft, den Kopf freizubekommen. (Foto: Beeldredactie Radboud University)

Beweis stellen kann. Selbstredend ist auch jemand, der oder die im Ruhrgebiet aufwächst und in Bonn studiert, sehr selbstständig. Dennoch befindet man sich immer noch innerhalb Deutschlands. Studiert man hingegen im Ausland, sieht man sich neben den ,normalen“ Problemen noch mit ganz anderen konfrontiert. Selbstständigkeit in einem fremden Land suggeriert, dass man in der Lage ist, sich anzupassen. An völlig fremde Umgebungen, eine andere Kultur und nicht zuletzt an den Umgang mit der anderen Sprache. Die einfachsten Dinge, wie der Besuch im Supermarkt, können einen vor Herausforderungen stellen. Lesen Arbeitgeber nun vom Auslandsstudium des Bewerbers, gehen sie davon aus, dass man auch diese gemeistert hat. Hinzu kommt die im Angesicht der Globalisierung zunehmende Bedeutung der Internationalität. Und ganz ehrlich: Wir gehen auch ins Ausland, weil wir es wollen. Man macht Erfahrungen, lernt ganz einfach die verschiedensten Leute kennen, kommt in Kontakt mit einer anderen Kultur, kann seine Unabhängigkeit unter Beweis stellen.

Es gibt also ausreichend Gründe ins Ausland zu gehen. Warum aber sollte man sich dann für die Niederlande entscheiden? Zunächst einmal sind die meisten unserer Studienbücher englischsprachig, weil sich eine Übersetzung ins Niederländische nicht lohnen würde. Wenn man sich nun vor Augen führt, dass die Mathematik ein sehr internationales Fach ist, wird der Vorteil deutlich. Unabhängig davon, ob man nach dem Studium in der Wissenschaft oder in der freien Wirtschaft tätig ist, die vorherrschende Sprache wird Englisch sein. An meiner Uni bin ich also von Anfang an mit mathematischem Englisch in Berührung gekommen. Durch diesen frühen Kontakt fühlt es sich ganz natürlich an, wenn man zum Beispiel Untersuchungsberichte auf englisch liest oder einen englischsprachigen Gastdozenten hat.

Was in meinem Fall den Ausschlag gegeben hat, ist der angenehme Rahmen. Dies äußert sich vor allem in der geringen Größe des Studienganges. Wenn ich in den Vor- lesungssaal komme, sitzen da nur etwa hundert andere Studierende. Und das ist schon zu Anfang des ersten Semesters so. Selbst in den Fächern, die man mit den Physikern und Informatikern zusammen hat, sitzt man mit höchstens 200 Menschen im Saal. Hierdurch entsteht eine fast schon familiäre Atmosphäre. Auch die Übungen finden in kleinen Gruppen statt, in denen auf 15 Studenten mindestens ein Assistent kommt. So hat dieser auch wirklich die Zeit, jede Frage in Ruhe zu beantworten. Es ist immer gut zu wissen, dass man einen persönlichen Ansprechpartner hat. Meine Universität sieht das genauso. Deshalb wird dafür gesorgt, dass der Kontakt zum Studentenberater hergestellt wird. Weiterhin wird uns in Kleingruppen im ersten Jahr ein Mentor zugeteilt, der uns hilft, den Übergang von der Schule zum Studium zu meistern. Außerdem gibt es immer auch eine Einführungswoche, in der man mehrere Mentoren zugewiesen bekommt.

Das sind alles Dinge, über die man im Voraus nachdenken sollte. Aber am Ende ist natürlich das Bauchgefühl entscheidend. Man kann sich viel erzählen lassen, sich im Internet informieren, oder Kontakt zur Studentenberatung aufnehmen. Die Hauptsache ist meiner Meinung nach, dass man dann noch zu einem Tag der offenen Tür oder zum Schnupperstudium geht. Dann kann man sich vor Ort überzeugen, dass die Universität wirklich zu einem passt. Generell gilt das für alle Universitäten, aber insbesondere auch für solche im Ausland. Das macht die Niederlande zu einem so geeigneten Ort für das Auslandsstudium. Durch die Nähe zu Deutschland ist es möglich, vor der Einschreibung den Campus zu besuchen. Auch eventuell aufkommendes Heimweh lässt sich besser bewältigen als in weiter Ferne.

Persönlich habe ich meine Wahl nie bereut. Tatsächlich kann ich mir ein Studium in Deutschland gar nicht mehr vorstellen. Das Land, die Menschen, die Sprache und nicht zuletzt meine Universität, die Radboud University: Auf nichts davon würde ich mehr verzichten wollen.

Leoni Winschermann, Stationsstraat I, AA 6678 Oosterhout, Nederland. leoniwinschermann@web.de

Leoni Winschermann hat 2015 ihre allgemeine Hochschulreife erhalten. Zusammen mit ihrem Zeugnis erhielt sie außerdem den DMVAbiturpreis. So kam sie erstmals mit der Vereinigung in Berührung. Danach ging sie an die Radboud University in Nijmegen, um ihr Mathematikstudium zu beginnen. Inzwischen ist sie im dritten Semester des Bachelorstudienganges Mathematik.

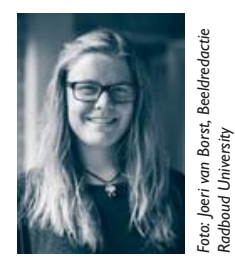

